\title{
The Tobacco Trichome Exudate Z-abienol and Its Relationship With Plant Resistance to Phytophthora nicotianae
}

\author{
William T. Steede, Justin M. Ma, David P. Eickholt, Katherine E. Drake-Stowe, and Sheri P. Kernodle, Crop and Soil Science Depart- \\ ment, North Carolina State University, Raleigh; H. David Shew, Department of Plant Pathology, North Carolina State University, Raleigh; \\ David A. Danehower, Avoca Inc., Merry Hill, NC; and Ramsey S. Lewis, Crop and Soil Science Department, North Carolina State University,
} Raleigh

\begin{abstract}
In previous research, we discovered a favorable quantitative trait locus (QTL) in cigar tobacco cultivar 'Beinhart 1000' designated as Phn15.1, which provides a high level of partial resistance to the black shank disease caused by Phytophthora nicotianae. A very close genetic association was also found between Phn15.1 and the ability to biosynthesize Z-abienol, a labdanoid diterpene exuded by the trichomes onto above-ground plant parts, and that imparts flavor and aroma characteristics to Oriental and some cigar tobacco types. Because accumulation of $Z$-abienol is considered to be undesirable for cultivars of other tobacco types, we herein describe a series of experiments to gain insight on whether this close association is due to genetic linkage or pleiotropy. First, in an in vitro bioassay, we observed $Z$-abienol and related diterpenes to inhibit hyphal growth of $P$. nicotianae at concentrations between 0.01 and $100 \mathrm{ppm}$. Secondly, we field-tested

transgenic versions of Beinhart 1000 carrying RNAi constructs for downregulating NtCPS2 or NtABS, two genes involved in the biosynthesis of $Z$-abienol. Thirdly, we also field tested a recombinant inbred line population segregating for a truncation mutation in $N t C P S 2$ leading to an interrupted $Z$-abienol pathway. We observed no correlation between field resistance to $P$. nicotianae and the ability to accumulate $Z$-abienol in either the transgenic materials or the mapping population. Results suggest that, although $Z$-abienol may affect $P$. nicotianae when applied at high concentrations in in vitro assays, the compound has little effect on black shank disease development under natural field conditions. Thus, it should be possible to disassociate Phn15.1-mediated black shank resistance identified in cigar tobacco cultivar Beinhart 1000 from the ability to accumulate Z-abienol, an undesirable secondary metabolite for burley and flue-cured tobacco cultivars.
\end{abstract}

Tobacco (Nicotiana tabacum L.), an economically-important Solanaceous crop that is cultivated worldwide, is susceptible to disease caused by a full range of plant pathogens. The most important disease affecting tobacco in many parts of the world is black shank disease caused by the oomycete Phytophthora nicotianae. This pathogen infects the roots and stems of the tobacco plant to cause stunting of plant development and ultimately plant death. Large-scale crop loss can result under favorable environmental conditions coupled with high inoculum densities.

Tobacco breeders have the objective of using natural or induced genetic variation to reduce economic loss from plant pathogens. Multiple mechanisms of genetic resistance or tolerance to $P$. nicotianae have been investigated and utilized in the development of new tobacco cultivars. This includes monogenic resistance to race 0 of $P$. nicotianae conferred by the $P h p$ and $P h l$ genes that were introgressed into $N$. tabacum from $N$. plumbaginifolia and N. longiflora, respectively (Apple 1962; Chaplin 1962; Johnson et al. 2002; Valleau et al. 1960); a very high level of apparent partial resistance conferred by a genomic region designated as $\mathrm{Wz}$ that was introgressed from N. rustica (Drake and Lewis 2013; Drake et al. 2015); and varying levels of polygenic partial resistance derived from materials such as Florida 301 (Xiao et al. 2012) or Beinhart 1000 (Vontimitta and Lewis 2011, 2012). Resistance conferred by $P h p, P h l$, and possibly $W z$ can be overcome by race shifts in pathogen populations. Appropriate breeding strategies are therefore those where single-gene resistance mechanisms

Corresponding author: Ramsey S. Lewis; E-mail: ramsey_lewis@ ncsu.edu

The authors declare no conflict of interest.

*The $\boldsymbol{e}$-Xtra logo stands for "electronic extra" and indicates that one supplementary figure is available online.

Accepted for publication 14 March 2017.

C 2017 The American Phytopathological Society are combined with genetic systems conferring medium to high levels of partial resistance.

A number of studies have been carried out to better understand the genetic control and underlying mechanisms of partial resistance to black shank in tobacco. Resistance in Florida 301 has been found to be controlled by multiple QTL with varying effects (Xiao et al. 2012). The cigar tobacco cultivar Beinhart 1000 exhibits an exceptionally high level of black shank resistance that has also been found to be controlled by multiple QTL with varying effects. A major QTL on linkage group 7, designated as Phn7.1, was found to exhibit large partial effects in mapping populations derived from crosses involving either Florida 301 or Beinhart 1000 (Vontimitta and Lewis 2011, 2012; Xiao et al. 2012). A major QTL on linkage group 15, designated as Phn15.1, was identified in a Beinhart $1000 \times$ Hicks mapping population, but not in a Florida $301 \times$ Hicks population (Vontimitta and Lewis 2011; Xiao et al. 2012). The Phn15.1 QTL may mostly explain the greater level of black shank resistance exhibited by Beinhart 1000 relative to Florida 301, and this allelic variability may be of great value for increasing the level of black shank resistance in U.S. flue-cured and burley tobacco.

To be of practical use in flue-cured and burley tobacco cultivar development, however, breeders would need to be able to transfer the favorable Phn15.1 allele from cigar tobacco cultivar Beinhart 1000 into elite breeding material without sacrificing yield and/or quality characteristics, including cured leaf chemistry. In the mapping studies of Vontimitta et al. (2010) and Vontimitta and Lewis (2011), the Phn15.1 QTL was found to be associated with two genes strongly influencing the accumulation of certain trichome exudates on aboveground parts of Oriental and some cigar tobacco cultivars. One gene, designated as BMVSE, is involved with the biosynthesis of Groups III through VI sucrose esters (Gwynn et al. 1985). The second gene, previously designated as $A b l$ by Tomita et al. (1980), is very closely associated with Phn15.1 and has recently been shown to encode for copalyl diphosphate synthase (Sallaud et al. 2012), one of two genes directly involved in the biosynthesis of the labdane diterpene $Z$-abienol from geranylgeranyl diphosphate (GGPP) (Supplementary Fig. S1). This gene was designated as NtCPS 2 by Sallaud et al. (2012). Functioning $B M V S E$ or NtCPS2 genes are not desired for flue-cured or burley 
tobacco cultivars, as compounds derived from the corresponding biosynthetic steps negatively affect flavor and aroma of cured leaf for these market classes.

$B M V S E$, the gene controlling the accumulation of groups III through VI sucrose esters, can be segregated away from the Phn15.1 (unpublished information). To date, however, the favorable Beinhart 1000 black shank resistance allele at $P h n 15.1$ has not been separated from the active NtCPS2 gene responsible for $Z$-abienol accumulation. The close association between these two traits could be due to either close genetic linkage (which should permit for separation of the two traits) or pleiotropy, in which the gene controlling $Z$-abienol accumulation (NtCPS2) also leads to the production of a chemical that interferes with $P$. nicotianae infection.

It is not unreasonable to hypothesize that $Z$-abienol, or a closely related molecule or derivative, may be involved in plant-pathogen interactions. Solanaceous plants exude a diversity of plant secondary products onto the surfaces of plant parts that may play roles in interactions with micro-organisms and herbivores (Cheng et al. 2007). For example, Nicotiana leaf surface diterpenes (including Z-abienol and the related molecule sclareol) have been reported to have activity against a range of fungi, oomycetes, bacteria, and disease development (Bailey et al. 1974; Cruickshank et al. 1977; Cohen et al. 1983; Kennedy et al. 1992, 1995; Menetrez et al. 1990; Seo et al. 2012; Shepherd and Mandryk 1963). Seo et al. (2003) suggested that the tobacco Z-abienol-related diterpene (11E, 13E)-labda-11,13-diene$8 \alpha, 15$-diol functions as an activator of stress response mechanisms. Fujimoto et al. (2015) showed that sclareol could cause inhibition of nematode penetration of roots of Arabidopsis and tomato, and that the compound may be an elicitor-like substance that activates defense responses.

The objectives of this research were to determine if $Z$-abienol, or a related molecule derived from the same diterpene pathway, might be associated with tobacco resistance to $P$. nicotianae. First, we utilized an in vitro assay system to investigate the effect of $Z$-abienol and several other related plant leaf surface exudates or diterpene compounds on hyphal growth of $P$. nicotianae. Second, because the last two downstream genes involved in the biosynthesis of $Z$-abienol, copalyl diphosphate synthase (NtCPS2) and Z-abienol synthase $(N t A B S)$, have been isolated and described (Sallaud et al. 2012), transgenic tobacco lines carrying RNAi interference constructs designed to downregulate expression of these genes were evaluated in field black shank nurseries for potentially altered susceptibility to $P$. nicotianae. Finally, a gene-specific single nucleotide polymorphism (SNP) encoding for a premature stop codon in $N t C P S 2$ was tested for its association with increased field black shank susceptibility in a recombinant inbred line (RIL) mapping population segregating for this mutation. The results of these experiments have implications for the potential to successfully incorporate the favorable Phn15.1 black shank resistance QTL into elite flue-cured and burley tobacco germplasm without sacrificing quality characteristics.

\section{Materials and Methods}

In vitro bioassay of inhibitory effects of leaf surface compounds on P. nicotianae. Preparative isolation of leaf surface compounds of interest. A total of seven leaf surface compounds or compound mixtures were tested for their inhibitory effects against $P$. nicotianae (Table 1). Labdene-diol, sclareolide, and a sclareol:13-epi-sclareol mixture were obtained as gifts from Avoca Inc. (Merry Hill, NC). The remaining four compounds were prepared via extraction of $205 \mathrm{~kg}$ of axillary buds of field-grown N. tabacum cultivar Galpao Comum.

A Patterson Double Cone Extractor (Scarborough, Canada) was used to carry out a $15 \mathrm{~min}$ extraction of the collected plant material in 1,000 liters of methanol. The methanol was then decanted and filtered using a crude filtration step, followed by filtration through a 25 micron filter. The filtered methanol extract was transferred to an APV Paraflash Vacuum Still (Tonawanda, NY) and distilled to result in $\sim 40$ liters of a residual dark brown liquid that was stored at $-4^{\circ} \mathrm{C}$ until evaporated in vacuo using a laboratory rotary evaporator. The crude extract was stored in amber bottles at $0^{\circ} \mathrm{C}$ until needed for further steps.

Fractionation of chemical components was carried out through preparative chromatography of the crude extract using a $2.5 \times$ $100 \mathrm{~cm}$ column of Sephadex LH-20 (GE Healthcare Biosciences, Sweden). Ten milliliters of a $300 \mathrm{mg} \mathrm{ml}^{-1}$ solution of the crude extract were loaded onto the column and eluted at $5 \mathrm{ml} \mathrm{min}^{-1}$ with a 97:3 methylene chloride/methanol mobile phase. One hundred and thirty-two $5 \mathrm{ml}$ fractions were collected, followed by one final $500 \mathrm{ml}$ collection. After each run, the column was washed with 1 liter of $95: 3$ methylene chloride/methanol at $5 \mathrm{ml} \mathrm{min}^{-1}$ and reequilibrated with 97:3 methylene chloride/methanol. The composition of individual test tube fractions or grouped fractions was determined using thin-layer chromatography on silica gel layers and via previously described gas chromatographic methods (Vontimitta et al. 2010). Verification of the identity of the compounds of interest (groups III through VI sucrose esters, $Z$-abienol, $\alpha$-cembratrienediol [ $\alpha$-CBTdiol], and $\beta$-cembratrienediol [ $\beta$-CBTdiol]) was carried out by comparison of retention times to that of authentic standards and/or confirmed by GC-MS analysis using an HP 5890 GC equipped with an HP5972 series MSD detector as previously outlined by Vontimitta et al. (2010).

Preparative separation of the Sephadex LH-20 gel fraction containing $Z$-abienol was carried out on a $2.5 \times 21.5 \mathrm{~cm}$ column of Merck Silica Gel 60 (Whitehouse Station, NJ). Two hundred milligrams of sample were loaded in a $1 \mathrm{ml}$ injection volume onto the column and eluted at a flow rate of $5 \mathrm{ml} \mathrm{min}^{-1}$ with a 4:1 methylene chloride/hexane mobile phase. Eighty-eight $5 \mathrm{ml}$ test tube fractions were collected. The column was washed with $100 \%$ methylene chloride and re-equilibrated with 4:1 methylene chloride/hexane. Individual test tube fractions were further analyzed via gas chromatography or thin layer chromatography.

Preparative chromatography of $\alpha$-CBTdiol and $\beta$-CBTdiol was carried out on $2.5 \times 21.5 \mathrm{~cm}$ bed of Merck Silica Gel 60. Two hundred milligrams of the combined Sephadex LH-20 fractions containing the diols were loaded onto the column in a $1 \mathrm{ml}$ injection volume, and the column was eluted at $5 \mathrm{ml} / \mathrm{min}$ with $1: 1: 8$ isopropanol/ chloroform/hexane. The first $20 \mathrm{~min}$ of eluate were collected as waste, followed by the collection of $445 \mathrm{ml}$ fractions. The column was washed with 1:1 methanol/methylene chloride and this wash, containing sucrose ester natural products, was collected. The column was then re-equilibrated with 1:1:8 isopropanol/chloroform/hexane. Chemical composition of the individual test tube fractions or grouped fraction collections was determined as described above.

Recrystallization of purified compounds was carried out according to Severson et al. (1985, 1988). Approximately one gram of $\alpha$-CBTdiol was dissolved in a minimum amount of pentane, filtered through a PTFE membrane into a vial, and the volume was reduced to 10 to $15 \mathrm{ml}$. The vial was capped and placed into a freezer $\left(-20^{\circ} \mathrm{C}\right)$.

Table 1. Nicotiana diterpenoids and related compounds tested for inhibition of pathogen hyphal growth

\begin{tabular}{ll}
\hline Compound & Common name \\
\hline (12Z)-labda-12, 14-dien-8 $\alpha$-ol & Z-abienol \\
$\alpha$-2,7,11-cembratriene-4,6-diol & $\alpha$-CBTdiol \\
$\beta-2,7,11$-cembratriene-4,6-diol & $\beta$-CBTdiol \\
(13E)-labda-13-ene-8 $\alpha, 15-d i o l$ & labdene-diol \\
(13R)-labda-14-ene-8,13-diol plus 13-epi-sclareol mixture & sclareol mixture \\
(3aR)-(+)-Sclareolide & sclareolide \\
(6-O-acetyl-2,3,4-tri-O-acyl)- $\alpha$-D-glucopyranosyl- $\beta$-D-fructofuranoside & sucrose esters \\
\hline
\end{tabular}


Crystals were allowed to form and filtered and washed with cold pentane. Yield of crystalline $\alpha$-CBTdiol was approximately 0.6 grams. Four hundred milligrams of the $\beta$-CBTdiol fraction was dissolved in a minimum amount of hot pentane. That volume was cooled in a freezer at $-20^{\circ} \mathrm{C}$ and the resulting crystals were collected, washed with cold pentane, and dried to yield $200 \mathrm{mg} \beta$-CBTdiol. Partially purified $Z$-abienol was dissolved in a minimum amount of acetone and deionized water was added to the cloud point. The solution was cooled to $-20^{\circ} \mathrm{C}$ and crystals were collected, washed with cold 3:1 acetone/deionized water, and dried under $\mathrm{N}_{2}$. The sucrose esters exist as six distinct groupings, groups I through VI, and are not generally subject to further purification. The SEs were isolated in sufficient purity as the column wash from the second chromatographic isolation of the CBT-diols.

Bioassay. The capacity of the compounds of interest to inhibit the growth of $P$. nicotianae was investigated via a bioassay that utilized a minimal nutrient solution consisting of one-tenth strength potato dextrose broth (PDB) adjusted to a $\mathrm{pH}$ of 5.1. This minimal media was used to limit nutrient supply, which could have potentially masked any inhibitory effects of the compounds of interest.

Initial leaf surface compound stocks were prepared in dimethyl sulfoxide (DMSO) at a concentration of $4 \mathrm{mg} \mathrm{ml}^{-1}$. Serial dilutions of this stock were then made to produce five final concentrations for testing: $0.01,0.1,1.0,10$, and $100 \mathrm{ppm}$. The commercial fungicide Ridomil Gold (Syngenta, Basel, Switzerland) was used as positive control. Pure DMSO was utilized as a negative control.

Experiments were performed using six-well Costar cell culture plates (Corning Costar, Corning, NY) with a volume of $16.8 \mathrm{ml}$ $\left(9.5 \mathrm{~cm}^{2}\right.$ cell growth area) per cell. Two milliliters of sterile PDB media were first dispensed into each well. Fifty microliters of the leaf surface compound dilutions in DMSO were added to the appropriate wells and mixed. The final concentration of DMSO per well was $2.5 \%$ of the total cell volume. Sterile \#2 cork borers were used to obtain agar plugs from the leading edge of active growing pathogen colonies and were placed in the center of each well. The plates were incubated at $28^{\circ} \mathrm{C}$ for 3 days. The entire colony was carefully removed after the incubation period and placed onto a tared aluminum weight vessel using a spatula. Vessels were dried at $70^{\circ} \mathrm{C}$ for $3 \mathrm{~h}$ and reweighed after equilibrating to room temperature. A control well containing the growing colony and DMSO without any leaf surface chemicals was present on each six-well plate, along with each of the five serial dilution concentrations.

The experiment was analyzed as a split-plot design, with main plots consisting of the chemical of interest and subplots consisting of the chemical concentration. The experiment was replicated over time with three replications. Data were analyzed using PROC MIXED (SAS Institute, Cary, NC) according to Littell et al. (1996). An F-test was first used to test for significant differences $(P<0.05)$ between subplots for pathogen weight. Because we were making preplanned comparisons, ESTIMATE statements were subsequently used to statistically compare, within each chemical treatment, the mean pathogen weight for each chemical concentration to the pathogen weight for the control (DMSO without added chemical).

Field evaluation of tobacco lines genetically engineered for reduced $Z$-abienol accumulation. We also had the objective of using metabolic engineering to investigate whether or not the ability to exude $Z$-abienol correlated with tobacco plant resistance to $P$. nicotianae under field conditions. In order to produce versions of cigar tobacco cultivar Beinhart 1000 that do not accumulate Z-abienol, Beinhart 1000 was transformed with RNAi constructs engineered to down-regulate expression of either NtABS or NtCPS 2. NtCPS 2 encodes a class-II terpene synthase that synthesizes 8-hydroxy-copalyl diphosphate from geranylgeranyl diphosphate, the substrate for initial biosynthesis of probably most diterpenes in plants, while NtABS encodes a kaurene synthase like protein that uses 8-hydroxy-copalyl diphosphate to produce $Z$-abienol (Sallaud et al. 2012).

The RNAi-based antisense constructs were generated in the vector pKYLX71 in a manner similar to that described by Siminszky et al. (2005). Two RNAi constructs were produced for silencing NtCPS2:
NtCPS2 2-1 and NtCPS2 4-6. For NtCPS2 2-1, a 135-bp segment was amplified from the $3^{\prime}$ region. To facilitate subsequent cloning steps, primers were designed to add a HindIII and XhoI site on the ends of the sense arm (5'-AGAAGCTTCCTCAAGCAACATCAAC CAAGACATTAAGCAAAC- $3^{\prime}$ and $3^{\prime}$-AAGTGTATAGGTTTCAC GAAAAAGTCGTTCAGATCGAGCTCTG-5') and a XbaI and SacI site on the ends of the antisense arm (5'-AGTCTAGACCTCAAG CAACATCAACCAAGACATTAAGCAAAC-3' and $3^{\prime}$-AAGTGTA TAGGTTTCACGAAAAAGTCGTTCAGATCCTCGAGTC-5'). Each

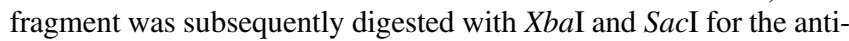
sense insertion, and with HindIII and $\mathrm{XhoI}$ for the sense insertion. The NtCPS2 2-1 sense fragment was subcloned into pKYLX80, followed by insertion of the antisense fragment. This intermediate vector enables the cloning of the sense and antisense fragments separated by a 151-bp soybean $\omega$-3 fatty acid desaturase intron to enhance stability of the construct in bacteria as well as increasing the efficiency of silencing in the plant. The NtCPS2 2-1 cassette from pKYLX80 was then digested with HindIII and SacI and ligated into the plant expression vector pKYLX71 to produce the final antiNtCPS2 2-1 RNAi construct. The pKYLX71 RNAi construct contains the Cauliflower mosaic virus (CaMV) 35S promoter that drives expression of the anti-NtCPS2 2-1 cassette and neomycin phosphotransferase II (nptII) gene to allow for selection of transformed plants in the presence of kanamycin. The same methodology was used to prepare the NtCPS2 4-6 and NtABS RNAi constructs. A 172-bp segment of $N t C P S 2$ designated 4-6 was amplified using primers for the sense arm (5'-GCAAGCTTTGCAACTTTCAACAATTTGG-3' and $3^{\prime}$-ACTAC TTCGGTGTTGAACCTGAGCTCAG-5') and antisense arm (5'-CAT CTAGATGCAACTTTCAACAATTTGG- ${ }^{\prime}$ and $3^{\prime}$-ACTACTTCGG TGTTGAACCTCTCGAGTC-5'). A 101-bp segment of NtABS was amplified using primers for the sense arm (5'-GCAAGCTTGTGATAT TATTAGATGCATGGACGAA- ${ }^{\prime}$ and $3^{\prime}$-GATACCCTTTTTGAAT GAATGTGACGAGAGCTCAG-5') and antisense arm (5'-CATCTA GAGTGATATTATTAGATGCATGGACGAA-3' and 3'-GATACCC TTTTTGAATGAATGTGACGACTCGAGTC-5' ${ }^{\prime}$ ).

The pKYLX71 RNAi constructs were introduced into Agrobacterium tumefaciens strain LBA4404. A doubled haploid (DH) approach was used to produce transgenic tobacco lines carrying the RNAi construct. Haploid Beinhart 1000 plants were produced using the method of Burk et al. (1979) and haploid leaf tissue transformed with the pKYLX71 NtABS RNAi or NtCPS2 RNAi constructs according to the procedure of An et al. (1986). Regenerated transgenic haploids were chromosome doubled using the midvein culture method of Kasperbauer and Collins (1972) to produce DH lines homozygous for the RNAi transgene insertion(s). Single primary transformants $\left(\mathrm{R}_{0}\right.$ generation) were self-pollinated in a greenhouse to produce $R_{1}$ seed.

Based upon a small-scale, preliminary evaluation of 40 transgenic doubled haploid lines during 2015, six NtABS RNAi and three NtABS RNAi versions of Beinhart 1000 were selected for further experimentation on the basis of their ultra-low levels of $Z$-abienol accumulation (data not shown). To generate replicated leaf surface chemistry data on these materials during the 2016 field growing season, 12 plants of each RNAi line, along with 12 plants each of control lines Beinhart 1000 , K326, K346, and Hicks, were evaluated in a disease-free environment for accumulation of the labdene diterpene $Z$-abienol and the cembranoid diterpenes $\alpha$-CBTdiol and $\beta$-CBTdiol. Plants were sampled and analyzed for these compounds using gas chromatography as previously described by Vontimitta et al. (2010). Levels of $Z$-abienol and total CBTdiols for the transgenic doubled haploid lines were compared with the non-trangsenic control, Beinhart 1000, using $t$ tests. For statistical tests, censored observations (those below the minimum limit of detection) were replaced by one-half the detection limit according to Atwood et al. (1991).

Also in 2016, these materials were evaluated for field black shank resistance in two naturally infested $P$. nicotianae soil-borne nurseries located at the Upper Coastal Plain Research Station near Rocky Mount, NC, and the Lower Coastal Plain Research Station near Kinston, NC. The experimental design at each location was a randomized complete block design with eight replications. Plots consisted of single, 12-plant rows with inter-row and within-row spacing of $1.14 \mathrm{~m}$ 
and $0.56 \mathrm{~m}$, respectively. Starting at approximately 40 days after transplanting, the number of plants killed by black shank was recorded. This continued throughout the growing season at intervals of approximately 14 days. At the end of the growing season, area under the disease progress curve (AUDPC) was calculated for each plot according to Madden et al. (2007). An overall analysis of variance was first carried out on the entire data set. Because we were interested in preplanned statistical comparisons (resistance of RNAi lines versus the non-transgenic control), genotypic AUDPC means for the transgenic lines were then compared with the mean of the nontransgenic control (Beinhart 1000) using single degree of freedom contrasts.

Testing the association between field black shank resistance and the presence/absence of an active $Z$-abienol biosynthetic pathway. To further investigate a possible association between field black shank resistance and $Z$-abienol accumulation, we also studied a mapping population segregating for the presence/absence of an active $Z$-abienol biosynthetic pathway. Tobacco accession TI1068, which accumulates $Z$-abienol, was hybridized with flue-cured tobacco cultivar K346, which does not accumulate $Z$-abienol because of an SNP (G $\rightarrow$ T transversion at position 292) in exon four of $N t C P S 2$, resulting in a truncated protein and disrupted biosynthesis of $Z$-abienol (Sallaud et al. 2012). A population consisting of $180 \mathrm{~F}_{4: 5}$ RILs derived from this cross was evaluated for black shank incidence at the Lower Coastal Plain Research Station near Kinston, NC, and the Upper Coastal Plain Research Station near Rocky Mount, NC, during 2014 and 2015 (for a total of four environments). The experimental design for each environment was a $10 \times 18$ alpha-lattice with three replications. The two parents were also randomized within each block for a total of 20 entries per block. Experimental units consisted of single $7 \mathrm{~m}$ long, 12-plant plots. Approximately 40 days after transplanting, the number of plants killed by black shank was recorded. This evaluation continued throughout the growing season at intervals of approximately 14 days. At the end of the growing season, AUDPC was calculated for each plot according to Madden et al. (2007).

AUDPC data for the RIL population were subjected to a square root transformation to reduce heterogeneity of error variances. Transformed data were used to obtain a best linear unbiased prediction (BLUP) for each RIL across all locations/years for each disease using ASReml version 3 (Gilmour et al. 2009). A mixed linear model was fit with random effects for RIL, environment, RIL $\times$ environment, replication within environment, and block within replication/environment. Each $\mathrm{F}_{4: 5}$ RIL was also genotyped, using a bulk of DNA collected from 10 plants per family, using a TaqMan marker assay previously described by Sallaud et al. (2012) to detect the $\mathrm{G} \rightarrow \mathrm{T}$ transversion at position 292 in exon four of NtCPS2. Subsequent marker-trait analyses were carried out using the BLUPs. The association between the K346 deleterious NtCPS2 truncation mutation and black shank AUDPC was tested using simple linear regression in R (R Core Team 2013).

\section{Results}

In vitro bioassay of inhibitory effects of leaf surface compounds on tobacco $P$. nicotianae. $P$. nicotianae hyphal growth was significantly $(P<0.01)$ inhibited at the $100 \mathrm{ppm}$ dosage levels of $\alpha$-CBTdiol, the sclareol mixture, sclareolide, and sucrose esters (Fig. 1). $Z$-abienol was found to significantly $(P<0.05)$ reduce growth of $P$. nicotianae at the $0.01,0.1,1$, and 100 ppm treatment levels. Inhibition by $Z$-abienol was marginally significant $(P=0.0556)$ at the 10 ppm concentration. No chemical treatment was as severe on P. nicotianae development as that of the positive control, Ridomil Gold.

Field evaluation of tobacco lines genetically engineered for reduced $\boldsymbol{Z}$-abienol accumulation. A total of six Beinhart 1000 $N t C P S 2$ RNAi doubled haploid lines and three NtABS RNAi lines were selected for field evaluation of resistance to disease caused by $P$. nicotianae. Both RNAi mechanisms were highly and significantly $(P<0.0001)$ effective at reducing levels of $Z$-abienol in field-grown plants (Fig. 2), leading to the production of nondetectable, or near nondetectable, levels of this leaf surface diterpene. Interestingly, levels of total CBTdiols (total of $\alpha$ - and $\beta$-CBTdiols) were decreased by an average of nearly $50 \%$ in the NtCPS2 RNAi lines relative to the non-transgenic control line (Fig. 2). More interestingly, levels of tobacco CBTdiols were significantly $(P<0.0001)$ reduced by greater than $90 \%$ in transgenic NtABS RNAi lines.

The large effects of the RNAi mechanisms made these transgenic doubled haploid lines an ideal system for directly testing the role, if any, of $Z$-abienol and potential derivatives, on field black shank resistance. Evidence of a high amount of disease pressure in the disease environments is indicated by the high amount of disease development in varieties K326 and Hicks, moderately and highly susceptible varieties, respectively. Although the accumulation of $Z$-abienol was reduced to nearly zero in the transgenic lines, black shank disease development in this material was not significantly affected $(P>0.05)$ (Fig. 2).

Testing the association between field black shank resistance and the presence/absence of an active $Z$-abienol biosynthetic pathway. The RIL mapping population derived from a cross between high, $Z$-abienol-accumulating germplasm accession TI1068 and nonaccumulating variety $\mathrm{K} 346$ provided an additional opportunity to test the relationship between an active $Z$-abienol biosynthetic pathway and black shank resistance. Genotyping of the $N t C P S 2 \mathrm{SNP}$ responsible for the difference in $Z$-abienol accumulation between the two parental lines (Sallaud et al. 2012) revealed no significant $(P=0.4483)$ association between the presence of an active $Z$-abienol biosynthetic pathway and black shank resistance (Fig. 3). Indeed, the homozygous wild-type genotypic class exhibited a slightly numerically greater level of disease development, although this difference was not significant.

\section{Discussion}

A number of previously published papers have indicated possible roles of tobacco diterpenes as plant defense stimulating molecules (Fujimoto et al. 2015; Seo et al. 2003), or as defense-related compounds per se (Bailey et al. 1974; Cruickshank et al. 1977; Cohen et al. 1983; Kennedy et al. 1992, 1995; Menetrez et al. 1990; Shepherd and Mandryk 1963). Previous trait mapping experiments in $N$. tabacum revealed a very strong genetic association between partial resistance to $P$. nicotianae conferred by QTL Phn15.1 and the ability of $N$. tabacum to accumulate the diterpene $Z$-abienol (Vontimitta et al. 2010; Vontimitta and Lewis 2012). The finding that the Z-abienol biosynthesis gene NtCPS2 cosegregates with Phn15.1 (Sallaud et al. 2012; Vontimitta and Lewis 2011) provided an intriguing possibility that $Z$-abienol, or a related molecule, may actually affect resistance to $P$. nicotianae. Experiments described in the current paper were motivated by an interest to gain insight on whether or not the strong relationship between the two traits is due to close physical linkage of genes controlling $Z$-abienol accumulation and partial resistance to $P$. nicotianae, or due to pleiotropy where an active $Z$-abienol biosynthesis gene is the actual gene underlying the Phn15.1 partial black shank resistance QTL. This is not only of high interest to those interested in the role of terpene compounds in plant defense, but also to those interested in improving tobacco varietal resistance to $P$. nicotianae. The favorable Beinhart 1000 allele at the Phn15.1 locus has a large effect on field black shank and represents novel genetic variability not currently present in flue-cured and burley tobacco germplasm (Vontimitta et al. 2010; Xiao et al. 2012). Transfer of this favorable allele from cigar tobacco to flue-cured and burley tobacco could substantially improve soil-borne disease resistance if it could be incorporated with minimal corresponding negative effects on yield and or cured leaf quality. Z-abienol results in a type of leaf chemistry not desired for flue-cured or burley tobacco, however. If the favorable Phn15.1 effect on black shank resistance is due to pleiotropic effects of the $Z$-abienol biosynthesis gene per se, it would not be possible to transfer the gene with a favorable effect on black shank resistance without simultaneously transferring an undesirable type of leaf chemistry.

In this investigation, an in vitro assay was first utilized to evaluate the inhibitory effect of selected diterpene compounds, including $Z$-abienol, and sucrose esters on hyphal development of $P$. nicotianae. Growth of $P$. nicotianae was somewhat negatively affected by $100 \mathrm{ppm}$ treatments of $\alpha$-CBTdiol, the sclareol mixture, sclareolide, sucrose esters, and moderately affected by $Z$-abienol. Although the 
observed inhibitory effects were generally observed only at the highest concentration levels, these results provided preliminary support for the possibility that $Z$-abienol, or a related labdane diterpene, may actually affect field resistance to the soil-borne pathogen P. nicotianae.

The mechanism of action of labdane diterpenes against fungal or fungal-like organisms is not fully understood. It has been hypothesized that these lipophilic compounds may be involved in membrane disruption (Cowan 1999), although their potential role in stimulation of defense responses cannot be excluded (Fujimoto et al. 2015; Seo et al. 2003). Stimulation of plant defense is not a possible mechanism that can be determined in the in vitro bioassay used in our experiments, however. Black shank is primarily a root and stem disease. We have no knowledge on the possibility that $Z$-abienol or related diterpenes may be exuded by tobacco roots. If not, there may be limited potential for physical interactions between the soil-borne pathogen $P$. nicotianae and $Z$-abienol, known to be a leaf and stem surface exudate. However, stalk trichome exudates might be expected to accumulate
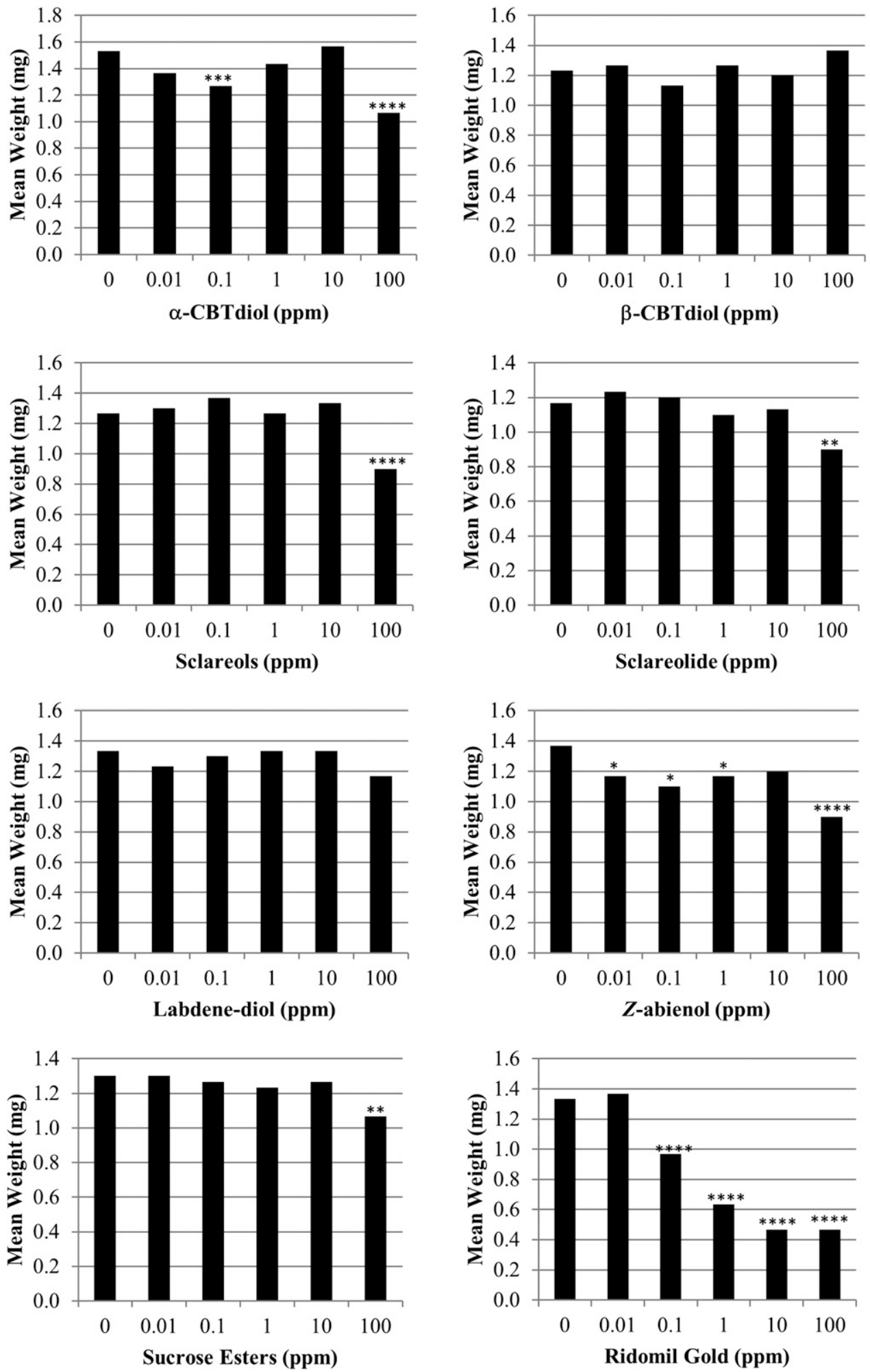

Fig. 1. Bioassay for inhibition of Phytophthora nicotianae by isolated leaf surface compounds. ${ }^{*},{ }^{* *},{ }^{* *}$, and ${ }^{* * *}$ indicate significant difference from the control at the $P<0.05,0.01$, 0.001 , and 0.0001 levels of significance, respectively. 
at the base of the tobacco stalk, a site of possible $P$. nicotianae infection. It is also possible that rain may wash leaf surface trichome exudates off of the plant and into the soil, where they could provide an inhibitory effect against $P$. nicotianae or other oomycetes.

Recent discovery of the specific genes involved in N. tabacum biosynthesis of Z-abienol (Sallaud et al. 2012) permitted for direct testing of the possibility that $Z$-abienol, or a related molecule, affects field resistance to $P$. nicotianae. First, transgenic versions of tobacco cultivar Beinhart 1000 were developed that expressed RNAi constructs designed to reduce expression of either NtABS or NtCPS2, the genes involved in the last and immediately preceding steps in the biosynthesis of $Z$-abienol from GGPP. RNAi was extremely
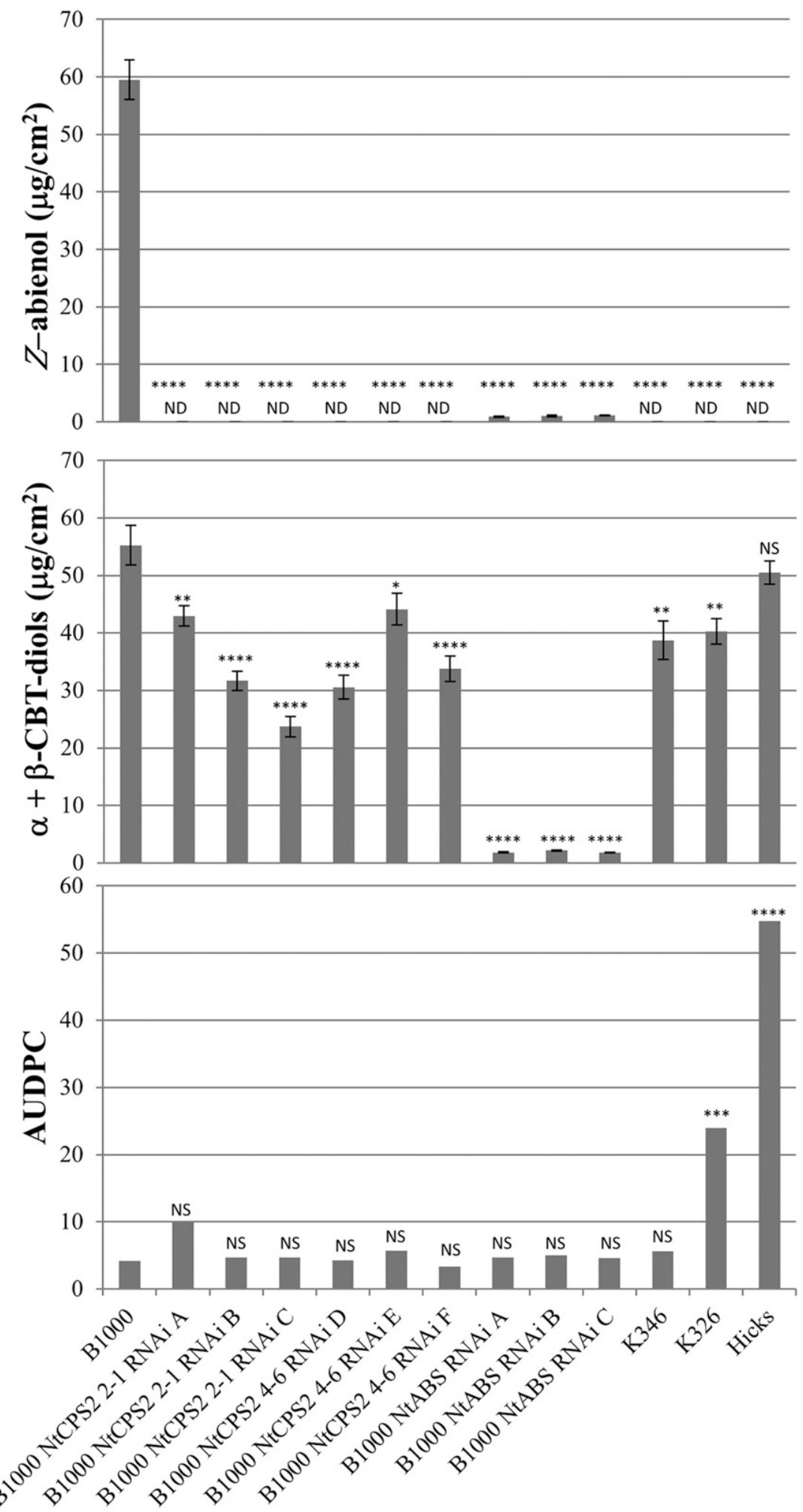

Fig. 2. Leaf surface chemistry determinations and field black shank resistance as measured by area under disease progress curve (AUDPC) for transgenic Beinhart 1000 (B1000) RNAi lines and associated control lines. $\mathrm{ND}=$ not detectable. Standard errors are provided for Z-abienol and total CBT average determinations. For leaf chemistry traits, genotypic means were compared with the nontransgenic control (Beinhart 1000) using $t$ tests. For AUDPC, genotypic means were compared with the nontransgenic control using single degree of freedom contrasts. ${ }^{*},{ }^{* *},{ }^{* * *}$, and ${ }^{* * * *}$ indicate significant difference from the control at the $P<0.05, P<0.01, P<0.001$, and $P<0.0001$ levels of significance, respectively. 'NS' indicates not significantly different from the control $(P>0.05)$. 
effective at reducing levels of $Z$-abienol on the leaf surface to nondetectable or near nondetectable amounts. Surprisingly, we observed a corresponding modest decrease in levels of the $\alpha$ - and $\beta$-CBTdiols in the NtCPS 2 lines, where a modest increase might have been expected because of reduced competition for the common substrate GGPP. In contrast, a $25 \%$ increase in the CBTdiols was previously observed in a mapping population segregating for a naturally occurring mutation in NtCPS2 (Vontimitta et al. 2010). Even more surprising was the tremendous decrease in the $\alpha$ - and $\beta$-CBTdiols after silencing NtABS. There is not sufficient nucleotide similarity (less than 10\%) between the sequences used in the NtABS RNAi constructs and either of the two genes involved in the biosynthesis of the CBTdiols from GGPP (cembratriene-ol synthase and $C Y P 71 D 16$ ) to permit for an expected simultaneous down-regulation of expression of either of these genes. Perhaps the buildup of 8-hydroxy-copalyl diphosphate that would be expected in the NtABS RNAi lines might operate in a feedback inhibition system to downregulate either of the two above-mentioned genes involved in the CBTdiol pathway. Nevertheless, the transgenic lines provided for an ideal system to test the potential role of $Z$-abienol or related labdane diterpenes on plant resistance to $P$. nicotianae under field conditions. This replicated testing revealed no significant reductions in field resistance to $P$. nicotianae in any of the RNAi lines. Indeed, the levels of disease development in the RNAi lines were largely equivalent to that of the untransformed control.

The association between field resistance to $P$. nicotianae and naturally occurring variability in NtCPS2 that cosegregates with Phn15.1 was also tested. Because deleterious mutations in NtCPS2 compromise the ability of $N$. tabacum to accumulate $Z$-abienol, a mapping population segregating for such a mutation permits investigation of a relationship between $Z$-abienol biosynthetic capacity and field black shank resistance. Similar to the transgenic doubled haploid RNAi lines, neither a numerical or significant relationship was observed between the two traits. The study of the RNAi lines and the mapping population should also provide insight on the question as to whether or not the molecule $(11 E, 13 E)$-labda-11,13-diene- $8 \alpha$, 15-diol (also designated as WAF-1 and suggested to be involved in plant defense by Seo et al. [2003]) might also be associated with field resistance to $P$. nicotianae. This compound is likely derived from labdene-diol (Sallaud et al. 2012), an intermediate whose accumulation should have been interrupted in the CPS2 RNAi lines. Although WAF1 was not quantified in the studies reported here, the lack of correlation between $Z$-abienol accumulation and field resistance to $P$. nicotianae does not indicate a strong role for WAF-1 in resistance to black shank.

Taken altogether, the results reported here suggest that $Z$-abienol can significantly inhibit $P$. nicotianae development in in vitro assays at levels of between 0.01 and $100 \mathrm{ppm}$. At levels present under field situations, however, $Z$-abienol has no apparent role on resistance to $P$. nicotianae. Overall, this leads one to conclude that partial

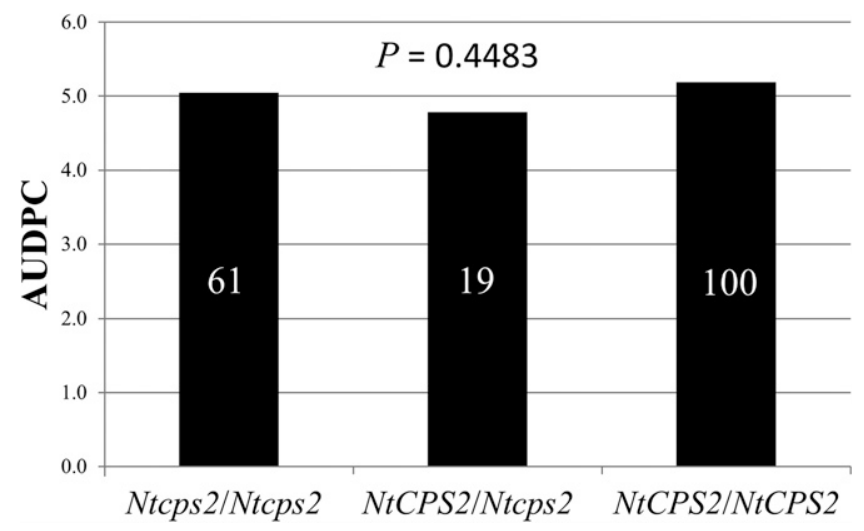

Fig. 3. NtCPS2 genotypic class means for black shank disease development for the K346 $\times$ TI1068 recombinant inbred line population as measured by area under disease progress curve (AUDPC). Numbers within bars indicates the number of families within each genotypic class. The $P$-value for testing the significance of the marker-trait association via linear regression is indicated above the histogram.
P. nicotianae resistance conferred by the favorable allele at Phn15.1 is not encoded for by NtCPS2, but rather a closely linked gene. Thus, if large populations are used, it should be possible to utilize marker assisted selection and backcrossing to transfer the favorable Phn15.1 allele from the cigar tobacco cultivar Beinhart 1000 to flue-cured and burley tobacco genotypes while selecting against the capacity to accumulate $Z$-abienol.

\section{Literature Cited}

An, G., Watson, B. D., and Cheng, C. C. 1986. Transformation of tobacco, tomato, potato, and Arabidopsis thaliana using a binary Ti vector system. Plant Physiol. 81:301-305.

Apple, J. L. 1962. Transfer of resistance to black shank (Phytophthora parasitica var nicotianae) from Nicotiana plumbaginifolia to N. tabacum. Phytopathology (Abstr.) 52:1.

Atwood, C. L., Blackwood, L. G., Harris, G. A., and Loehr, C. A. 1991 Recommended methods for statistical analysis of data containing less-thandetectable measurements. EGG-SARE-9247, Rev. 1. Idaho National Engineering Laboratory, EG\&G Idaho, Inc., Idaho Falls, ID. Available at https://www.osti. gov/scitech/biblio/6174750. doi:10.2172/6026384

Bailey, J. A., Vincent, G. G., and Burden, R. S. 1974. Diterpenes from Nicotiana glutinosa and their effects on fungal growth. J. Gen. Microbiol. 85:57-64.

Burk, L. G., Gerstel, D. U., and Wernsman, E. A. 1979. Maternal haploids of Nicotiana tabacum L. from seed. Science 206:585.

Chaplin, J. F. 1962. Transfer of black shank resistance from Nicotiana plumbaginifolia to flue-cured N. tabacum. Tob. Sci. 6:184-189.

Cheng, A.-X., Lou, Y.-G., Mao, Y.-B., Lu, S., Wang, L.-J., and Chen, X.-Y. 2007. Plant diterpenoids: biosynthesis and ecological functions. J. Integr. Plant Biol. 49:179-186.

Cohen, Y., Eyal, H., Goldschmidt, Z., and Sklarz, B. 1983. A preformed chemical inhibitor of tobacco powdery mildew on the leaves of Nicotiana glutinosa Physiol. Plant Pathol. 22:143-150.

Cowan, M. M. 1999. Plant products as antimicrobial agents. Clin. Microbiol. Rev. 12:564-582.

Cruickshank, I. A. M., Perrin, D. R., and Mandryk, M. 1977. Fungitoxicity of duvatrienediols associated with the cuticular wax of tobacco leaves. Phytopathol. Z. 90:243-249.

Drake, K. E., and Lewis, R. S. 2013. An introgressed Nicotiana rustica genomic region confers resistance to Phytophthora nicotianae in cultivated tobacco. Crop Sci. 53:1366-1374.

Drake, K. E., Moore, J. M., Bertrand, P., Fortnum, B., Peterson, P., and Lewis, R. S. 2015. Black shank resistance and agronomic performance of flue-cured tobacco lines and hybrids carrying the introgressed Nicotiana rustica region, Wz. Crop Sci. 55:79-86.

Fujimoto, T., Mizukubo, T., Abe, H., and Seo, S. 2015. Sclareol induces plant resistance to root-knot nematode partially through ethylene-dependent enhancement of lignin accumulation. Mol. Plant-Microbe Interact. 28:398-407.

Gilmour, A. R., Gogel, B. J., Cullis, B. R., and Thompson, R. 2009. ASReml User Guide Release 3.0. VSN International Ltd, Hemel Hempstead, U.K.

Gwynn, G. R., Severson, R. F., Jackson, D. M., and Stephenson, M. G. 1985. Inheritance of sucrose esters containing $\beta$-methylvaleric acid in tobacco. Tob. Sci. 29:79-81.

Johnson, E. S., Wolff, M. F., Wernsman, E. A., Atchley, W. R., and Shew, H. D. 2002. Origin of the black shank resistance gene, $P h$, in tobacco cultivar Coker 371-Gold. Plant Dis. 86:1080-1084.

Kasperbauer, M. A., and Collins, G. B. 1972. Reconstitution of diploids from leaf tissue of anther-derived haploids in tobacco. Crop Sci. 12:98-101.

Kennedy, B. S., Nielsen, M. T., Severson, R. F., Sisson, V. A., Stephenson, M. K., and Jackson, D. M. 1992. Leaf surface chemicals from Nicotiana affecting germination of Peronospora tabacina (Adam). sporangia. J. Chem. Ecol. 18:1467-1479.

Kennedy, B. S., Nielson, M. T., and Severson, R. F. 1995. Biorationals from Nicotiana protect cucumbers against Colletotrichum lagenarium (Pass.) Ell. \& Halst disease development. J. Chem. Ecol. 21:221-231.

Littell, R. C., Milliken, G. A., Stroup, W. W., and Wolfinger, R. D. 1996. SAS system for mixed models. SAS Institute. Inc., Cary, NC.

Madden, L. V., Hughes, G., and van der Bosch, F. 2007. The Study of Plant Disease Epidemics. APS Press, St. Paul, MN.

Menetrez, M. L., Spurr, H. W., Jr., Danehower, D. A., and Lawson, D. R. 1990 Influence of tobacco leaf surface chemicals on germination of Peronospora tabacina Adam sporangia. J. Chem. Ecol. 16:1565-1576.

R Core Team. 2013. R: A language and environment for statistical computing. R Foundation for Statistical Computing, Vienna, Austria, http://www.R-project.org/.

Sallaud, C., Giacalone, C., Topfer, R., Goepfert, S., Bakaher, N., Rosti, S., and Tissier, A. 2012. Characterization of two genes for the biosynthesis of the labdane diterpene Z-abienol in tobacco (Nicotiana tabacum) glandular trichomes. Plant J. 72:1-17.

Seo, S., Gomi, K., Kaku, H., Abe, H., Seto, H., Nakatsu, S., Neya, M., Kobayashi, M., Nakaho, K., Ichinose, Y., Mitsuhara, I., and Ohashi, Y. 2012. Identification of natural diterpenes that inhibit bacterial wilt disease in tobacco, tomato, and Arabidopsis. Plant Cell Physiol. 53:1432-1444.

Seo, S., Seto, H., Koshino, H., Yoshida, S., and Ohashi, Y. 2003. A diterpene as an endogenous signal for the activation of defense responses to infection with Tobacco mosaic virus and wounding in tobacco. Plant Cell 15:863-873. 
Severson, R. F., Arrendale, R. F., Chortyk, O. T., Green, C. R., Thome, F. A., Stewart, J. L., and Johnson, A. W. 1985. Isolation and characterization of the sucrose esters of the cuticular waxes of green tobacco leaf. J. Agric. Food Chem. 33:870-875.

Severson, R. F., Stephenson, M. G., Johnson, A. W., Jackson, D. M., and Chortyk, O. T. 1988. Isolation and preparative chromatography of the major cuticular diterpenes of green tobacco. Tob. Sci. 32:99-103.

Shepherd, C. J., and Mandryk, M. 1963. Germination of conidia of Peronospora tabacina (Adam): Germination in vivo. Aust. J. Biol. Sci. 16:77-87.

Siminszky, B., Gavilano, L., Bowen, S. W., and Dewey, R. E. 2005. Conversion of nicotine to nornicotine in Nicotiana tabacum is mediated by CYP82E4, a cytochrome P450 monooxygenase. Proc. Natl. Acad. Sci. USA 102: 14919-14924.

Tomita, H., Sato, M., and Kawashima, N. 1980. Inheritance of labdanoid producing ability in Nicotiana tabacum. Agric. Biol. Chem. 44:2517-2518.
Valleau, W. D., Stokes, G. W., and Johnson, E. M. 1960. Nine years experience with the Nicotiana longiflora factor for resistance to Phytophthora parasitica var. nicotianae in the control of black shank. Tob. Sci. 4:92-94.

Vontimitta, V., Danehower, D. A., Steede, T., and Lewis, R. S. 2010. Analysis of a Nicotiana tabacum genomic region controlling two leaf surface chemistry traits. J. Agric. Food Chem. 58:294-300.

Vontimitta, V., and Lewis, R. S. 2011. Mapping of quantitative trait loci affecting resistance to Phytophthora nicotianae in tobacco (Nicotiana tabacum L.) line Beinhart-1000. Mol. Breed. 29:89-98.

Vontimitta, V., and Lewis, R. S. 2012. Growth chamber evaluation of a Nicotiana tabacum 'Beinhart 1000 ' $\times$ 'Hicks' mapping population for QTL affecting resistance to multiple races of Phytophthora nicotianae. Crop Sci. 52:91-98.

Xiao, B., Drake, K., Vontimitta, V., Tong, Z., Zhang, X., Li, M., Leng, X., and Lewis, R. S. 2012. Location of genomic regions contributing to Phytophthora nicotianae resistance in tobacco cultivar 'Florida 301.'. Crop Sci. 53:473-481. 